\title{
Highly nondegenerate four-wave mixing efficiency of an asymmetric coupled quantum well structure
}

\author{
Roberto Paiella and Kerry J. Vahala \\ Department of Applied Physics, Mail Stop 128-95, California Institute of Technology, \\ Pasadena, California 91125
}

(Received 8 November 1994; accepted for publication 28 February 1995)

\begin{abstract}
An asymmetric coupled quantum well structure is theoretically investigated as a means of tailoring the conversion efficiency of the four-wave mixing process at terahertz detuning rates. In this structure, a coherent electronic oscillation between the two wells can be excited that introduces a resonance peak in the four-wave mixing frequency response. A calculation based on the density matrix formalism shows that an increase in the power conversion efficiency on the order of $10 \mathrm{~dB}$ can be attained at the selected resonance frequency for low temperature operation. Finally, we propose a novel technique for exciting the interwell oscillations that takes advantage of the polarization dependence of the interband optical transitions in alternating strain quantum wells.@ 1995 American Institute of Physics.
\end{abstract}

Nondegenerate four-wave mixing (NDFWM) in semiconductor traveling wave amplifiers (TWAs) has attracted considerable interest in recent years, ${ }^{1-3}$ both because it provides direct information on the ultrafast nonlinear dynamics of semiconductor gain media, and because of its potential applications in lightwave communication systems. In particular, NDFWM devices are attractive candidates for use as broadband wavelength converters and for fiber dispersion compensation by optical phase conjugation. ${ }^{4-6}$

The physical mechanisms responsible for FWM in the semiconductor active layer are modulation of the carrier density by interband photomixing of the input pump and probe waves, and modulation of the occupation probability of each (sub)band caused by these same input waves but associated with various intraband processes (including spectral hole burning and carrier heating). The resulting dynamic gain and index grating partially scatters the input waves into new sideband signals. Carrier density pulsations are limited by the recombination lifetime $\tau_{s}$ typically on the order of $100 \mathrm{ps}$ in optical amplifiers; intraband processes are governed by much shorter $(<1 \mathrm{ps})$ relaxation times, so that, although their scattering efficiency is much smaller, they become the dominant mechanism at detuning frequencies in excess of $100 \mathrm{GHz} .{ }^{1-3}$

The objective of this letter is to provide theoretical evidence for the feasibility of engineering the ultrafast NDFWM frequency response by means of specially designed TWA structures, leading to an enhanced conversion efficiency at terahertz detuning rates. In particular, we propose a scheme for tailoring the NDFWM frequency response based on the asymmetric coupled quantum well (ACQW) structure shown in the inset of Fig. 1, which was originally proposed by Luryi $^{7}$ as a means to setting up coherent electric-dipole oscillations across a heterostructure barrier. ${ }^{8,9}$ Here, we propose a novel technique for exciting sustained tunnelingdipole oscillations, based on the use of alternating strain quantum wells, and show how these oscillations can be used to introduce an ultrafast resonance peak in the NDFWM frequency response.

The two quantum wells of the idealized structure of Fig. 1 are designed so that their ground states in the conduction band $(|l\rangle$ and $|r\rangle$ for the left and right well, respectively) would be degenerate with energy $E_{0}$ if the well separation were large enough to prevent state coupling. If the barrier is thin enough to allow coupling, however, the true stationary states of the composite system are given approximately by the bonding and antibonding combinations

$$
\begin{aligned}
& |b\rangle=\frac{1}{\sqrt{2}}(|l\rangle+|r\rangle), \quad E_{b}=E_{0}-\frac{\hbar \omega_{t}}{2}, \\
& |a\rangle=\frac{1}{\sqrt{2}}(|l\rangle-|r\rangle), \quad E_{a}=E_{0}+\frac{\hbar \omega_{t}}{2},
\end{aligned}
$$

where $\omega_{t}$ depends on the strength of the coupling. On the other hand, the single-well levels in the valence band are intentionally nondegenerate, so that coupling between them is negligible.

If an electron is initially localized in either well (i.e., is prepared in the coherent superposition of the conduction band stationary states corresponding to either single-well state), it will then oscillate across the barrier at frequency $\omega_{t}$, because of the different rates at which the phases of $|a\rangle$ and $|b\rangle$ evolve with time. In the work of Refs. 8 and 9, the required initial condition $\left(|\psi\rangle_{t=0}=|l\rangle\right.$ or $\left.|r\rangle\right)$ was accomplished by exciting the system with ultrashort light pulses of spectral width larger than $\omega_{t}$ and center frequency tuned to that of the desired quantum well transition $\left(\omega_{l}\right.$ or $\omega_{r}$ for the left or right well, respectively).

Alternatively, one could take advantage of the dipole transition selection rules in the presence of strain. Assume that tensile strain is introduced in the left well and compressive strain in the right well. Then, one can preferentially excite the single-well states of the left (right) well only, using TM (TE) polarized radiation (this technique has recently been used by Zhou et al. to probe interwell transport in alternating-strain TWAs $^{10}$ ).

In a FWM experiment under these conditions, the occupation probability of each well (whose modulation is responsible for the NDFWM process) behaves like a harmonic oscillator driven at the detuning frequency. A resonance peak is therefore introduced in the FWM frequency response at a detuning frequency equal to the "natural" frequency $\omega_{t}$ (typically a few terahertz). 


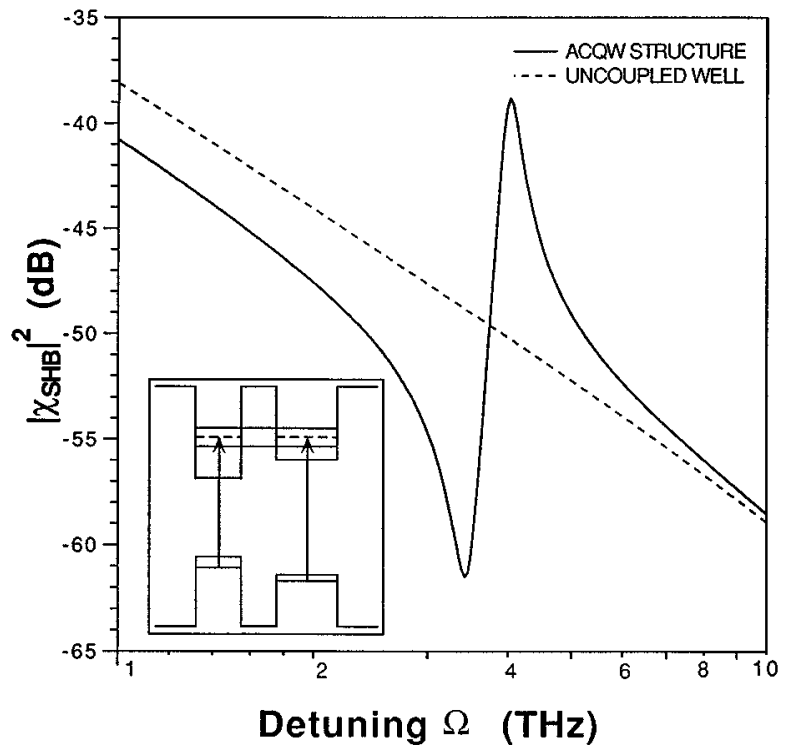

FIG. 1. Magnitude squared of the spectral-hole-burning contribution to $\chi^{\mathrm{FWM}}$ in the ACQW structure (schematically shown in the inset) vs detuning frequency, for $\tau_{1}=\tau_{d}=7 \mathrm{ps}$ (as in Ref. 9 at $10 \mathrm{~K}$ ), $\omega_{t}=4 \mathrm{THz}$. Here $\chi_{\mathrm{SHB}}$ is in arbitrary units. As explained in the text, in this detuning range $\left|\chi_{\mathrm{SHB}}\right|^{2}$ gives a good estimate of the overall FWM power conversion efficiency. For comparison, $\left|\chi_{\mathrm{SHB}}\right|^{2}$ in the limit of no interwell coupling is also shown (dotted line).

In the following analysis, the ACQW structure is described as a set of inhomogeneously broadened three-level systems labeled by the two-dimensional wave vector $k$ parallel to the junction plane. It is convenient to write the density matrix $\rho$ corresponding to each such system in the $\{|r\rangle,|l\rangle,|v\rangle\}$ representation $(|v\rangle$ being the ground state of the left well in the valence band), where only $|l\rangle$ and $|v\rangle$ are optically coupled (for TM waves). We consider the case of copropagating pump and probe waves, with the pump much stronger than any other signal; the total electric field is then given by

$$
\begin{aligned}
E(t)= & E_{p} \exp \left(-i \omega_{p} t\right)+E_{q} \exp \left(-i \omega_{q} t\right) \\
& +E_{s} \exp \left(-i \omega_{s} t\right)+\text { c.c. }
\end{aligned}
$$

where $p, q$, and $s$ denote pump, probe, and converted signal, respectively, and the detuning frequency is $\Omega=\omega_{p}-\omega_{q}$ $=\omega_{s}-\omega_{p}$. To calculate the induced optical polarization, we solve the equation of motion for the density matrix $\rho$ and use

$$
P(t)=\frac{1}{V} \sum_{k} \mu^{k}\left(\rho_{l v}^{k}+\rho_{v l}^{k}\right),
$$

where $V$ is the active region volume, $\mu$ is the electric dipole moment operator, and the superscripts denote dependence on $k$ and will be omitted henceforth. For simplicity we treat the case of only one subband.

The damping processes affecting the density matrix include spontaneous recombination, and carrier-carrier and carrier-phonon scattering. Relaxation due to scattering processes is best formulated in the $\{|a\rangle,|b\rangle,|v\rangle\}$ representation, since it involves relaxation towards quasiequilibrium Fermi distributions, which are properly defined only for the coupled-well energy eigenstates. In this basis, the collisional relaxation rate reads as follows:

$$
R_{\mathrm{coll}}=-\left(\begin{array}{ccc}
\frac{1}{\tau_{1}}\left(\rho_{a}-f_{a}\right) & \frac{1}{\tau_{d}} \rho_{a b} & \frac{1}{\tau_{2}} \frac{\rho_{a v}}{\sqrt{2}} \\
\frac{1}{\tau_{d}} \rho_{b a} & \frac{1}{\tau_{1}}\left(\rho_{b}-f_{b}\right) & \frac{1}{\tau_{2}} \frac{\rho_{b v}}{\sqrt{2}} \\
\frac{1}{\tau_{2}} \frac{\rho_{v a}}{\sqrt{2}} & \frac{1}{\tau_{2}} \frac{\rho_{v b}}{\sqrt{2}} & \frac{1}{\tau_{1}}\left(\rho_{v}-f_{v}\right)
\end{array}\right),
$$

where $1 / \tau_{d}$ is the rate at which the tunneling oscillations are damped, $1 / \tau_{1}$ is the rate at which a quasi-Fermi distribution $f$ is established in the $|a\rangle-|b\rangle$ states, and $1 / \tau_{2}$ is the optical dipole dephasing rate (which affects $\rho_{1 v}$ only; hence, the factors $2^{-1 / 2}$ ). $R_{\text {coll }}$ may be readily converted to the $\{|r\rangle,|l\rangle,|v\rangle\}$ representation using Eq. (1) and the definition of the density matrix. For the sake of simplicity, we omit in $R_{\text {coll }}$ and in the following analysis spontaneous recombination and carrier-phonon relaxation leading to dynamic carrier heating. These effects will be included in a more detailed treatment and are not central to the mechanism proposed here.

Solution of the equations of motion for the density matrix in the context of FWM is well documented in the literature. ${ }^{11-13}$ Following these approaches, and using a procedure similar to that of Ref. 7 to treat the interwell oscillations, the steady-state solution for $\rho_{1}$ is given by

$$
\begin{aligned}
\rho_{l}= & \frac{\bar{f}_{a}+\bar{f}_{b}}{2}+\operatorname{Re}\left[\left(\tau_{1}+\frac{\tau_{d}}{1+\omega_{t}^{2} \tau_{d}^{2}}\right) A\right. \\
& +\left(\frac{\tau_{1}}{1-i \Omega \tau_{1}}+\frac{1 / 2 \tau_{d}}{1-i\left(\Omega+\omega_{t}\right) \tau_{d}}\right. \\
& \left.\left.+\frac{1 / 2 \tau_{d}}{1-i\left(\Omega-\omega_{t}\right) \tau_{d}}\right) B e^{-i \Omega t}\right]
\end{aligned}
$$

where the overbar denotes the quasiequilibrium distributions determined by the pumping level, and where

$$
\begin{aligned}
& A=-\frac{\mu^{2}}{\hbar^{2}}\left(\frac{\bar{f}_{a}+\bar{f}_{b}}{2}-\bar{f}_{v}\right) \tilde{\chi}\left(\omega_{p}\right)\left|E_{p}\right|^{2}, \\
& B=-\frac{\mu^{2}}{\hbar^{2}}\left(\frac{\bar{f}_{a}+\bar{f}_{b}}{2}-\bar{f}_{v}\right)\left\{\left[\tilde{\chi}\left(\omega_{p}\right)+\tilde{\chi}^{*}\left(\omega_{q}\right)\right] E_{p} E_{q}^{*}\right. \\
& \left.+\left[\tilde{\chi}\left(\omega_{s}\right)+\tilde{\chi}^{*}\left(\omega_{p}\right)\right] E_{s} E_{p}^{*}\right\}, \\
& \tilde{\chi}(\omega)=\frac{\tau_{2}}{1-i\left(\omega-\omega_{l}\right) \tau_{2}} .
\end{aligned}
$$

In order to estimate the FWM conversion efficiency of the ACQW structure, we need to evaluate the harmonic component of the polarization density at $\omega_{s}$, which provides the source of the converted signal, and is given by ${ }^{12}$ 


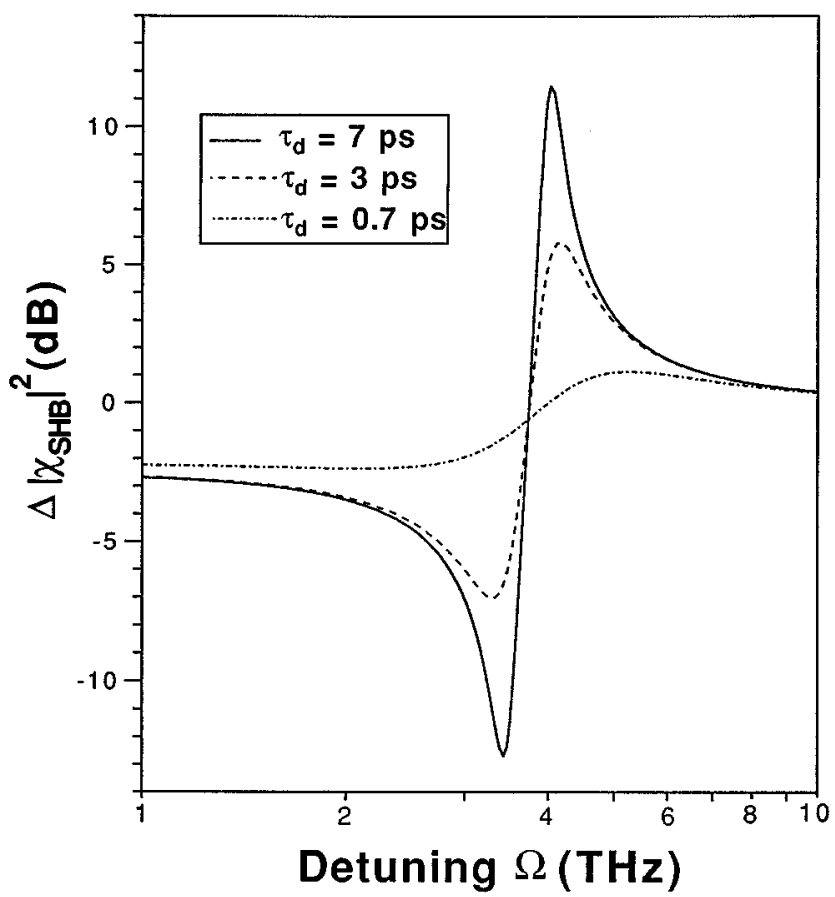

FIG. 2. $\left|\chi_{\mathrm{SHB}}\right|^{2}$ in the ACQW structure relative to its value in the uncoupled-well limit vs detuning frequency, for $\omega_{t}=4 \mathrm{THz}$ and several different values of $\tau_{d}$.

$$
\begin{aligned}
P_{s}= & \frac{1}{V} \sum_{k} \mu \rho_{l v}^{\left(\omega_{s}\right)} \\
= & -\frac{i}{V} \sum_{k} \frac{\mu^{2}}{\hbar} \tilde{\chi}\left(\omega_{s}\right)\left[\left(\rho_{l}^{(0)}-\rho_{v}^{(0)}\right) E_{s}\right. \\
& \left.+\left(\rho_{l}^{(\Omega)}-\rho_{v}^{(\Omega)}\right) E_{p}\right],
\end{aligned}
$$

where the superscripts distinguish among harmonics at different frequencies. Substituting $\rho_{l}^{(0)}$ and $\rho_{l}^{(\Omega)}$ as given in (5) and the similar expressions (but without the terms involving $\left.\omega_{t}\right)$ for $\rho_{v}^{(0)}$ and $\rho_{v}^{(\Omega)}, P_{s}$ can be cast in the general form

$$
P_{s}=\epsilon_{0} \chi^{0} E_{s}+\epsilon_{0} \chi^{\mathrm{FWM}} E_{p}^{2} E_{q}^{*},
$$

where the term proportional to $E_{q}^{*}$ is responsible for FWM, and in general has contributions from carrier density pulsations, spectral hole burning, carrier heating, and $\chi^{\mathrm{FWM}}$ $=\chi_{\mathrm{CDP}}+\chi_{\mathrm{CH}}+\chi_{\mathrm{SHB}} \cdot{ }^{12}$ At detuning frequencies on the order of $\omega_{t}\left(>1 / \tau_{1}\right.$ as assumed here $)$, spectral hole burning is expected to be the dominant FWM process, so that the magnitude of $\chi_{\mathrm{SHB}}$ is a good estimate of the overall FWM efficiency in this detuning range. As seen below, the interaction between the beating of the pump and probe waves and the tunneling oscillations enters directly in the spectral hole burning term by introducing a resonance at $\omega_{t}$, for both negative and positive detuning frequencies (second and third terms in parentheses),

$$
\begin{aligned}
\chi_{\mathrm{SHB}}= & \frac{i}{\epsilon_{0}}\left(\frac{3 / 2 \tau_{1}}{1-i \Omega \tau_{1}}+\frac{1 / 4 \tau_{d}}{1-i\left(\Omega+\omega_{t}\right) \tau_{d}}+\frac{1 / 4 \tau_{d}}{1-i\left(\Omega-\omega_{t}\right) \tau_{d}}\right) \\
& \times \frac{1}{V} \sum_{k} \frac{\mu^{4}}{\hbar^{3}}\left(\frac{\bar{f}_{a}+\bar{f}_{b}}{2}-\bar{f}_{v}\right) \tilde{\chi}\left(\omega_{s}\right)\left[\tilde{\chi}\left(\omega_{p}\right)+\tilde{\chi}^{*}\left(\omega_{q}\right)\right] .
\end{aligned}
$$

Note that in writing Eq. (9) we assumed conditions such that $\omega_{t}$ may be taken to be approximately independent of $k$.

The magnitude squared of $\chi_{\mathrm{SHB}}(\Omega)$, which is proportional to the power conversion efficiency, is plotted in Fig. 1 at terahertz detuning rates, for $\omega_{t}=4 \mathrm{THz}, \tau_{1}=\tau_{d}$ $=7 \mathrm{ps}$ (the value measured in a GaAs/AlGaAs ACQW structure at $10 \mathrm{~K}^{9}$ ), and assuming that $\tau_{2}$ is shorter than any other time constant. For comparison, $\left|\chi_{\mathrm{SHB}}\right|^{2}$ in the limit of no coupling between the wells is also shown (dotted line). A pronounced peak, preceded by a dip of comparable size, clearly appears at resonance, giving rise to a maximum increase in the conversion efficiency of more than $10 \mathrm{~dB}$ at $\Omega=\omega_{t}$. Note that at detuning frequencies below $\omega_{t},\left|\chi_{\mathrm{SHB}}\right|^{2}$ is smaller in the ACQW structure than in the uncoupled case by approximately $3 \mathrm{~dB}$, because the oscillations across the barrier reduce the (time-average) amplitude of the "spectral hole." However, the resulting decrease in the overall FWM efficiency becomes negligible at sub-terahertz detuning rates, where carrier heating and carrier density pulsations are dominant.

The size of the resonance peak is obviously limited by the damping rate $1 / \tau_{d}$. This is illustrated in Fig. 2 , where the change in $\left|\chi_{\mathrm{SHB}}\right|^{2}$ due to the coupling between the wells is plotted for different values of $\tau_{d}$. As shown in this figure, the effect of the interwell coupling should be detectable for $\tau_{d}$ as small as several hundred femtoseconds, indicating that higher temperature operation is possible. These results therefore substantiate the possibility of engineering the NDFWM frequency response by going beyond the standard uncoupled quantum-well TWAs. A new class of FWM devices may then be envisioned, with frequency response characteristics tailored to specific applications in terahertz spectroscopy and wavelength conversion.

This work was sponsored by the Office of Naval Research (N00014-91-J-1524) and the National Science Foundation (ECS-9412862).

${ }^{1}$ L. F. Tiemeijer, Appl. Phys. Lett. 59, 499 (1991).

${ }^{2}$ K. Kikuchi, M. Kakui, C. E. Zah, and T. P. Lee, IEEE J. Quantum Electron. 28, 151 (1992)

${ }^{3}$ J. Zhou, N. Park, J. W. Dawson, K. J. Vahala, M. A. Newkirk, and B. I. Miller, Appl. Phys. Lett. 63, 1179 (1993).

${ }^{4}$ M. C. Tatham, G. Sherlock, and L. D. Westbrook, IEEE Photonics Technol. Lett. 5, 1303 (1993).

${ }^{5}$ J. Zhou, N. Park, J. W. Dawson, K. J. Vahala, M. A. Newkirk, and B. I. Miller, IEEE Photonics Technol. Lett. 6, 50 (1994).

${ }^{6}$ M. C. Tatham, X. Gu, L. D. Westbrook, G. Sherlock, and D. M. Spirit, Electron. Lett. 30, 1335 (1994).

${ }^{7}$ S. Luryi, IEEE J. Quantum Electron. 27, 54 (1991).

${ }^{8}$ K. Leo, J. Shah, E. O. Göbel, T. C. Damen, S. Schmitt-Rink, W. Schäfer, and K. Köhler, Phys. Rev. Lett. 66, 201 (1991).

${ }^{9}$ H. G. Roskos, M. C. Nuss, J. Shah, K. Leo, D. A. B. Miller, A. M. Fox, S. Schmitt-Rink, and K. Köhler, Phys. Rev. Lett. 68, 2216 (1992).

${ }^{10}$ J. Zhou, N. Park, K. J. Vahala, M. A. Newkirk, and B. I. Miller, Appl. Phys. Lett. 65, 1897 (1994).

${ }^{11}$ G. P. Agrawal, J. Opt. Soc. Am. B 5, 147 (1988).

${ }^{12}$ A. Uskov, J. Mørk, and J. Mark, IEEE J. Quantum Electron. 30, 1769 (1994).

${ }^{13}$ J. Mørk and A. Mecozzi, Appl. Phys. Lett. 65, 1736 (1994). 